\title{
Integral equation and simulation studies of the Heisenberg spin fluid in an external magnetic field
}

\author{
F. Lado* and E. Lomba \\ Instituto de Química Física Rocasolano, CSIC, Serrano 119, 28006 Madrid, Spain
}

J. J. Weis

Laboratoire de Physique Théorique et Hautes Energies, Bâtiment 211, Université de Paris-Sud, 91405 Orsay Cedex, France

(Received 26 February 1998)

\begin{abstract}
We develop a general method to study inhomogeneous liquids in an external field using orthogonal polynomials tailored to the one-body density. The procedure makes integral equation calculations of these systems no more difficult than those of ordinary homogeneous molecular fluids. We apply this method to the ferromagnetic Heisenberg spin fluid in an external magnetic field using both the reference-hypernetted chain closure and a reference version of the Zerah-Hansen closure, with no further approximations. The calculation includes a mapping of the two-phase region for several values of the external magnetic field. Comparison with Monte Carlo simulation data shows the integral equation procedure yielding nearly exact results, in particular for nonzero external fields. [S1063-651X(98)15109-7]
\end{abstract}

PACS number(s): 61.20.Gy, 64.60.-i, 75.10.-b, 75.30.-m

\section{INTRODUCTION}

The Gibbsian $N$-body density function of a Hamiltonian $H_{N}$ that is rotationally and translationally invariant must itself be rotationally and translationally invariant, as must then also be all reduced $n$-body density functions of this Hamiltonian. In particular, the one-body density is a constant and the two-body density depends only on relative coordinates. An external field destroys this homogeneity, producing anisotropy or nonuniformity [1,2], and so makes necessary the joint calculation of the coupled one-body and two-body density functions. A striking if familiar example of the response of a bulk system to an external field is ferromagnetism. In this paper, we shall use this particular case to present a general procedure [3] to compute the coupled one-body and twobody density functions of an inhomogeneous classical fluid in an external field. Remarkably, the procedure is no more difficult to carry through than similar calculations for ordinary homogeneous systems.

Perhaps the simplest model of a disordered continuum system exhibiting ferromagnetic behavior is a fluid of hard spheres with embedded Heisenberg spins described using classical statistical mechanics [4-8]. On the one hand, the simplifications of this model compared to magnetic dipoledipole interactions are significant for simulation. The dipoledipole model presents substantial conceptual difficulties in connection with the nonexistence of the thermodynamic limit for orientationally ordered phases. Moreover, its simulation results are strongly dependent on the boundary conditions. Simulations for the Heisenberg spin model are free of these problems. (The integral equation formalism developed below serves equally well for either potential.) On the other hand,

\footnotetext{
*On leave from Department of Physics, North Carolina State University, Raleigh, NC 27695-8202.
}

disordered Heisenberg spin systems are of interest in their own right. It has been known for some time that $\mathrm{Co} / \mathrm{P}$ alloys [9] and $\mathrm{Co} / \mathrm{Au}$ melts [10] have a tendency to form amorphous ferromagnets, although their "true liquid" nature has been questioned because of the technical difficulties posed by undercooling below the Curie temperature. While these systems might be more representative of quenched spin fluids, very recently Albrecht and co-workers [11] have finally managed to undercool a $\mathrm{Co}_{80} \mathrm{Pd}_{20}$ melt below its Curie temperature at zero field, thus obtaining the first evidence of ferromagnetic behavior in a liquid metal in conditions where the Heisenberg exchange interaction absolutely dominates the magnetic dipole-dipole contribution. The exchange interaction is the crucial term in the present paper and it is our main aim to analyze how it models the phase behavior with and without the presence of external fields.

The Heisenberg spin fluid in an external magnetic field $\mathbf{B}_{0}$ is defined by the canonical partition function

$$
\begin{aligned}
Z= & \frac{1}{N ! \Lambda^{3 N}} \int \prod_{j=1}^{N}\left[d \mathbf{r}_{j} d \omega_{j}\right] \exp \left(\beta \sum_{j} \boldsymbol{\mu}_{j} \cdot \mathbf{B}_{0}\right. \\
& \left.-\beta \sum_{i<j} u_{0}\left(r_{i j}\right)-\beta \sum_{i<j} u_{s s}\left(r_{i j}, \omega_{i}, \omega_{j}\right)\right) .
\end{aligned}
$$

This can be factored into an ideal part and the excess, $Z$ $=Z^{\text {id }} Z^{\text {ex }}$, where

$$
\begin{aligned}
Z^{\mathrm{id}} & =\frac{1}{N ! \Lambda^{3 N}} \int \prod_{j=1}^{N}\left[d \mathbf{r}_{j} d \omega_{j}\right] \exp \left(\beta \sum_{j} \boldsymbol{\mu}_{j} \cdot \mathbf{B}_{0}\right) \\
& =\frac{1}{N !}\left(\frac{V}{\Lambda^{3}}\right)^{N}\left[4 \pi \frac{\sinh \beta \mu B_{0}}{\beta \mu B_{0}}\right]^{N},
\end{aligned}
$$




$$
\begin{aligned}
Z^{\mathrm{ex}}= & \frac{1}{(4 \pi V)^{N}} \int \prod_{j=1}^{N}\left[d \mathbf{r}_{j} d \omega_{j} f_{0}\left(\omega_{j}\right)\right] \\
& \times\left(-\beta \sum_{i<j} u_{0}\left(r_{i j}\right)-\beta \sum_{i<j} u_{s s}\left(r_{i j}, \omega_{i}, \omega_{j}\right)\right) .
\end{aligned}
$$

Here $\beta=1 / k_{B} T$ is the inverse Kelvin temperature, with $k_{B}$ Boltzmann's constant, $\Lambda$ the de Broglie thermal wavelength, and $\omega=(\theta, \phi)$ the orientation of the magnetic dipole moment $\boldsymbol{\mu}$, referred to the uniform field $\mathbf{B}_{0}$ as the $z$ direction. The interaction energies are written as pairwise sums of the hard sphere potential $u_{0}(r)$ for spheres of diameter $\sigma$ and the Heisenberg spin-spin potential $u_{s s}\left(r, \omega_{1}, \omega_{2}\right)$ for $r>\sigma$,

$$
\begin{gathered}
u_{s s}\left(r, \omega_{1}, \omega_{2}\right)=-J(r) \hat{\mathbf{s}}_{1} \cdot \hat{\mathbf{s}}_{2}, \\
\beta J(r)=K \frac{e^{-\kappa(r / \sigma-1)}}{r / \sigma},
\end{gathered}
$$

where $\hat{\mathbf{s}}$ is the unit spin vector in the direction of $\boldsymbol{\mu}$. In this last expression, the dimensionless coupling strength $K$ may be read as the inverse reduced temperature, $K=1 / T^{*}$, while $\kappa$ is a dimensionless range parameter. Finally, in Eq. (3), $f_{0}(\omega)$ is the normalized one-body orientational distribution of a noninteracting spin system,

$$
f_{0}(\omega)=\frac{e^{\beta \mu B_{0} \cos \theta}}{\sinh \left(\beta \mu B_{0}\right) / \beta \mu B_{0}} .
$$

From this starting point, we present here a complete study of the thermodynamics, structure, and magnetic properties of the topologically disordered Heisenberg spin fluid, with and without applied fields. We pay particular attention to the ferromagnetic region of the phase diagram, i.e., where the system undergoes spontaneous magnetization. We show how the tailored orthogonal polynomial technique presented in this paper casts the inhomogeneous problem of the orientationally ordered fluid into the familiar terms of a homogeneous molecular fluid. Thus we can resort to standard integral equation techniques, such as the reference hypernetted chain equation and the Zerah-Hansen equation, whose calculated results are then extensively compared with Monte Carlo simulation data. Our interest in the phase behavior extends to the gas-liquid transition and its coupling to the paramagnetic-ferromagnetic transition at zero field. (We are aware that the Heisenberg interaction is inadequate to model the low density behavior of ferrofluids, which is controlled by the longer range dipole-dipole interactions, but one might expect that the action of external fields on the gas-liquid equilibrium should be similar in both cases. Future work will concentrate on the dipole-dipole interaction.) In order to explore the gas-liquid transition, we have also performed Gibbs ensemble Monte Carlo (GEMC) simulations for the spin system in the presence of external magnetic fields, including a fully aligned system. The results agree remarkably well with the integral equation estimates. In this way we achieve a thorough description of the translationally disordered phases of our spin system: paramagnetic gas/liquid and ferromagnetic liquid, with good estimates for the Curie temperatures.
The rest of the paper proceeds as follows. In the next section we present a full account of the integral equation techniques devised to deal with the orientationally ordered phases of our system, including explicit expressions for the calculation of thermodynamic and magnetic properties. Details of the simulation procedures can be found in Sec. III, in particular the sampling techniques required for a correct probing of the orientational phase space in strongly anisotropic systems such as the ferromagnets here considered. Finally, an in-depth discussion of the most significant results for magnetic, thermodynamic, and structural properties is presented in Sec. IV.

\section{INTEGRAL EQUATION FORMULATION}

\section{A. One-body and two-body density functions}

The key quantities needed for a complete magnetic and thermodynamic description of this system are the one-body and two-body density functions,

$$
\begin{aligned}
& \rho^{(1)}(\mathbf{r}, \omega)=\left\langle\sum_{j=1}^{N} \delta\left(\mathbf{r}-\mathbf{r}_{j}\right) \delta\left(\omega-\omega_{j}\right)\right\rangle=\frac{\rho}{4 \pi} f(\omega) \\
& \rho^{(2)}\left(\mathbf{r}, \omega, \mathbf{r}^{\prime}, \omega^{\prime}\right) \\
& =\left\langle\sum_{i \neq j} \delta\left(\mathbf{r}-\mathbf{r}_{i}\right) \delta\left(\omega-\omega_{i}\right) \delta\left(\mathbf{r}^{\prime}-\mathbf{r}_{j}\right) \delta\left(\omega^{\prime}-\omega_{j}\right)\right\rangle \\
& =\frac{\rho^{2}}{(4 \pi)^{2}} f(\omega) f\left(\omega^{\prime}\right) g\left(\left|\mathbf{r}-\mathbf{r}^{\prime}\right|, \omega, \omega^{\prime}\right)
\end{aligned}
$$

where $\rho=N / V$ is the density and $f(\omega)$ the one-body orientational distribution in the interacting fluid. Equation (8) defines the generalized pair distribution function $g\left(r, \omega, \omega^{\prime}\right)$ of the inhomogeneous spin system in an external magnetic field. The angular brackets in these definitions denote a canonical ensemble average with the Hamiltonian of Eq. (1).

The basic equations that determine the distribution functions $f(\omega)$ and $g\left(r, \omega, \omega^{\prime}\right)$ are well known $[12,13]$. The onebody density can be differentiated with respect to $x=\cos \theta$ to give

$$
\begin{aligned}
\frac{d}{d x} \ln \left[\frac{f(\omega)}{f_{0}(\omega)}\right]= & -\frac{\rho}{4 \pi} \int d \mathbf{r} d \omega^{\prime} f\left(\omega^{\prime}\right) \\
& \times g\left(r, \omega, \omega^{\prime}\right) \frac{d \beta u_{s s}\left(r, \omega, \omega^{\prime}\right)}{d x},
\end{aligned}
$$

the first member of a Kirkwood-Born-Green-Yvon hierarchy [14]. Calculation of $f(\omega)$ from this equation requires knowing $g$. In classical liquid state theory, the pair distribution function is obtained from the Ornstein-Zernike (OZ) equation and a closure relation $[12,13]$. The first of these, generalized for anisotropy, reads

$$
\begin{aligned}
\gamma\left(r_{12}, \omega_{1}, \omega_{2}\right)= & \frac{\rho}{4 \pi} \int d \mathbf{r}_{3} d \omega_{3} f\left(\omega_{3}\right)\left[\gamma\left(r_{13}, \omega_{1}, \omega_{3}\right)\right. \\
& \left.+c\left(r_{13}, \omega_{1}, \omega_{3}\right)\right] c\left(r_{32}, \omega_{3}, \omega_{2}\right)
\end{aligned}
$$


for the indirect correlation function $\gamma=g-1-c$, where $c$ is the direct correlation function. The second, or closure, relation expresses $c$ back in terms of $\gamma$ and the model's pair interactions,

$$
\begin{aligned}
c\left(r, \omega_{1}, \omega_{2}\right)= & \exp \left[-\beta u_{0}(r)-\beta u_{s s}\left(r, \omega_{1}, \omega_{2}\right)\right. \\
& \left.+\gamma\left(r, \omega_{1}, \omega_{2}\right)+b\left(r, \omega_{1}, \omega_{2}\right)\right] \\
& -1-\gamma\left(r, \omega_{1}, \omega_{2}\right) .
\end{aligned}
$$

This relation must be supplemented with an approximation for $b$, the so-called bridge function, which is formally defined in terms of a diagram summation [12] that offers little practical benefit. Most approximate closures for $c$ define $b$ implicitly. We note that the derivative of $f(\omega)$ in Eq. (9) can alternatively be related to the direct correlation function $c$ through the Lovett-Mou-Buff-Wertheim equation $[15,16]$.

\section{B. Expansions in special orthogonal polynomials}

A practical approach to solving Eqs. (9)-(11) for any classical fluid in an external field using the same numerical procedures already employed for homogeneous systems was sketched in Ref. [3]. The essential ingredient turns out to be orthogonal polynomials tailored to the specific one-body distribution of the fluid [17]. The usual procedure for orientation-dependent functions such as $\gamma\left(r, \omega_{1}, \omega_{2}\right)$ is to expand in spherical harmonics $Y_{l m}(\omega)$ [18],

$$
\begin{gathered}
\gamma\left(r, \omega_{1}, \omega_{2}\right)=4 \pi \sum_{l_{1}, l_{2}, m} \gamma_{l_{1} l_{2} m}(r) Y_{l_{1} m}\left(\omega_{1}\right) Y_{l_{2} \bar{m}}\left(\omega_{2}\right), \\
Y_{l m}(\omega)=\frac{1}{\sqrt{4 \pi}}(-1)^{m} e^{i m \phi} P_{l m}(\cos \theta),
\end{gathered}
$$

where $P_{l m}(\cos \theta)$ is the familiar associated Legendre function, but here renormalized to two, and $\bar{m}=-m$. This expansion in fact makes the solution of the inhomogeneous liquid equations very difficult. We will instead expand in modified spherical harmonics,

$$
\begin{gathered}
\gamma\left(r, \omega_{1}, \omega_{2}\right)=4 \pi \sum_{l_{1}, l_{2}, m} \gamma_{l_{1} l_{2} m}(r) \mathcal{Y}_{l_{1} m}\left(\omega_{1}\right) \mathcal{Y}_{l_{2}} \bar{m}\left(\omega_{2}\right), \\
\mathcal{Y}_{l m}(\omega)=\frac{1}{\sqrt{4 \pi}}(-1)^{m} e^{i m \phi} \mathcal{P}_{l m}(\cos \theta) .
\end{gathered}
$$

The modified Legendre functions $\mathcal{P}_{l m}(\cos \theta)$ are explicitly constructed, beginning with $\mathcal{P}_{00}(\cos \theta)=1$, using the GramSchmidt method [19] with the orthonormality condition

$$
\frac{1}{2} \int_{-1}^{1} d x f(x) \mathcal{P}_{l m}(x) \mathcal{P}_{l^{\prime} m}(x)=\delta_{l l^{\prime}}
$$

where $f(\cos \theta)$ is the one-body distribution of the fluid. For an isotropic fluid with $f(x)=1$, this construction produces the standard Legendre functions $P_{l m}(x)$ (normalized to two). For an anisotropic fluid with $f(x) \neq 1$, the difference between $P_{l m}(x)$ and $\mathcal{P}_{l m}(x)$ is crucial.

To graphically illustrate this difference, we show in Fig. 1

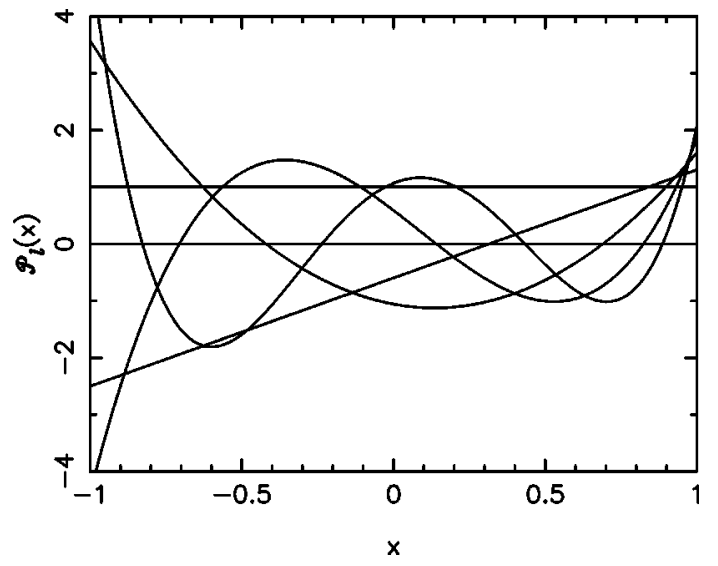

FIG. 1. Modified Legendre polynomials $\mathcal{P}_{l}(x) \equiv \mathcal{P}_{l 0}(x)$ (normalized to 2) generated by the Gram-Schmidt method for weight function $f_{0}(x)$ with $\beta \mu B_{0}=1$. The order $l$ of the polynomials can be read from the number of roots.

the first few modified Legendre polynomials $\mathcal{P}_{l 0}(x)$ obtained using the distribution $f_{0}(x)$, Eq. (6), with $\beta \mu B_{0}=1$, a small anisotropy. Except for $\mathcal{P}_{00}(x)$, the symmetry (antisymmetry) about $x=0$ of the standard polynomials $P_{l 0}(x)$ for $l$ even (odd) is lost in the presence of an external field, which here is in the direction $x=1$. Similarly asymmetric shapes are obtained for the $\mathcal{P}_{l m}(x)$ with $m>0$, the asymmetry gradually becoming less pronounced as $m$ increases. The calculation of the modified Legendre functions is described in Appendix A.

The numerical evaluation of the $\mathrm{OZ}$ equation is simpler in Fourier transform representation, which deconvolutes the $\mathbf{r}$ integral. Thus, in transform space, Eq. (10) becomes

$$
\begin{aligned}
\tilde{\gamma}\left(k, \omega_{1}, \omega_{2}\right)= & \frac{\rho}{4 \pi} \int d \omega_{3} f\left(\omega_{3}\right)\left[\tilde{\gamma}\left(k, \omega_{1}, \omega_{3}\right)\right. \\
& \left.+\widetilde{c}\left(k, \omega_{1}, \omega_{3}\right)\right] \tilde{c}\left(k, \omega_{3}, \omega_{2}\right),
\end{aligned}
$$

with one remaining integration. This final integral can also be eliminated and the evaluation of the $\mathrm{OZ}$ equation reduced to algebra by now expanding the pair functions as in (13); Eq. (15) then becomes

$$
\tilde{\gamma}_{l_{1} l_{2} m}(k)=(-1)^{m} \rho \sum_{l_{3}}\left[\tilde{\gamma}_{l_{1} l_{3} m}(k)+\tilde{c}_{l_{1} l_{3} m}(k)\right] \tilde{c}_{l_{3} l_{2} m}(k) .
$$

The significant feature here is that this $\mathrm{OZ}$ equation for an inhomogeneous fluid in an external field is identical to that of an ordinary homogeneous fluid [18] and so, along with some closure equation familiar from simple liquids, can be solved for $g\left(r, \omega_{1}, \omega_{2}\right)$ with the same algorithms already used for homogeneous systems [20,21].

\section{Numerical procedures}

Equations (9)-(11) being nonlinear, their solution is iterative. Given a set of approximate coefficients $\gamma_{l_{1} l_{2} m}(r)$, an improved set is produced by the following steps:

(1) Closure relation + Fourier transform: 


$$
\begin{aligned}
\gamma\left(r, x_{1}, x_{2}, y\right)= & \sum_{l_{1}, l_{2}, m} \gamma_{l_{1} l_{2} m}(r) \mathcal{P}_{l_{1} m}\left(x_{1}\right) \mathcal{P}_{l_{2} m}\left(x_{2}\right) \\
& \times(-1)^{m} T_{m}(y), \\
c\left(r, x_{1}, x_{2}, y\right)= & \exp \left[-\beta u_{0}(r)-\beta u_{s s}\left(r, x_{1}, x_{2}, y\right)\right. \\
& \left.+\gamma\left(r, x_{1}, x_{2}, y\right)+b\left(r, x_{1}, x_{2}, y\right)\right] \\
& -1-\gamma\left(r, x_{1}, x_{2}, y\right), \\
c_{l_{1} l_{2} m}(r)= & \frac{1}{4 \pi} \int_{-1}^{1} \frac{d y}{\sqrt{1-y^{2}}} \int_{-1}^{1} d x_{2} \int_{-1}^{1} d x_{1} \\
\times & f\left(x_{1}\right) f\left(x_{2}\right) c\left(r, x_{1}, x_{2}, y\right) \\
& \times \mathcal{P}_{l_{1} m}\left(x_{1}\right) \mathcal{P}_{l_{2} m}\left(x_{2}\right)(-1)^{m} T_{m}(y), \\
\tilde{c}_{l_{1} l_{2} m}(k)= & \frac{4 \pi}{k} \int_{0}^{\infty} d r r c_{l_{1} l_{2} m}(r) \sin k r .
\end{aligned}
$$

(2) OZ equation + inverse Fourier transform:

$$
\begin{aligned}
\tilde{\Gamma}_{m}(k)= & (-1)^{m} \rho \widetilde{C}_{m}(k) \widetilde{C}_{m}(k) \\
& \times\left[I-(-1)^{m} \rho \widetilde{C}_{m}(k)\right]^{-1}, \\
\gamma_{l_{1} l_{2} m}(r)= & \frac{1}{2 \pi^{2} r} \int_{0}^{\infty} d k k \tilde{\gamma}_{l_{1} l_{2} m}(k) \sin k r .
\end{aligned}
$$

In these equations, $x=\cos \theta, y=\cos \phi$, and $T_{m}(y)$ is a Chebyshev polynomial; further, in Eq. (21), $I$ is the unit matrix while $\widetilde{\Gamma}_{m}(k), \widetilde{C}_{m}(k)$ are symmetric matrices with elements $\tilde{\gamma}_{l_{1} l_{2} m}(k), \tilde{c}_{l_{1} l_{2} m}(k)$, respectively, with $l_{1}, l_{2} \geqslant m$. The inversion (19) is performed using $n$-point Gaussian quadratures with the zeroes of $\mathcal{P}_{n 0}(x)$ and $T_{n}(y)$. Upon convergence of the $\gamma_{l_{1} l_{2} m}(r)$ coefficients, the generalized pair distribution function $g$ is finally computed as

$$
\begin{aligned}
g\left(r, \omega_{1}, \omega_{2}\right)= & \exp \left[-\beta u_{0}(r)-\beta u_{s s}\left(r, \omega_{1}, \omega_{2}\right)\right. \\
& \left.+\gamma\left(r, \omega_{1}, \omega_{2}\right)+b\left(r, \omega_{1}, \omega_{2}\right)\right] .
\end{aligned}
$$

This solution for $g\left(r, \omega_{1}, \omega_{2}\right)$ is obtained using the polynomials $\mathcal{P}_{l m}(x)$ generated with the current $f(x)$. One now returns to Eq. (9) and updates the one-body distribution $f(x)$. In expanded form, this equation is

$$
\begin{gathered}
\frac{d}{d x} \ln \left[\frac{f(x)}{f_{0}(x)}\right]=\sum_{l_{1}, l_{2}, m} \xi_{l_{1} l_{2} m} \mathcal{P}_{l_{1} m}(x) \frac{d \mathcal{P}_{l_{2} m}(x)}{d x}, \\
\xi_{l_{1} l_{2} m}=-\rho \int d \mathbf{r} \sum_{l_{3}} g_{l_{1} l_{3} m}(r) u_{l_{3} l_{2} m}(r),
\end{gathered}
$$

where the $u_{l_{1} l_{2} m}(r)$ are the known coefficients (see below) of the spin-spin interaction $u_{s s}\left(r, \omega_{1}, \omega_{2}\right)$, so that finally

$$
\ln f(x)=\ln f_{0}(x)+\sum_{l=0}^{\infty} a_{l} \mathcal{P}_{l 0}(x)
$$

Here $a_{l}$ for $l>0$ is determined by numerical (Gaussian) integration of Eq. (24) and $a_{0}$ by normalization. The $n$-point Gaussian quadrature (see Appendix A) is exact for polynomial integrands of degree less than $2 n$, a condition that always holds for the computed $a_{l}$ coefficients. For the calculations reported in this paper, it turns out that the term linear in $x$ dominates the series expansion in Eq. (26), so that an effective field $B$ acting on a particle that includes the magnetic effects of the particle's surroundings through the coefficient $a_{1}$ can be calculated from this equation. In the absence of an interaction $\left(a_{1}=0\right)$ this field is of course just $B_{0}$.

The iterations for $f(\omega)$ and $g\left(r, \omega_{1}, \omega_{2}\right)$ are continued until both functions are self-consistently determined. The only approximation in the calculation is the unavoidable one in every liquid state calculation to date, the representation of the bridge function $b\left(r, \omega_{1}, \omega_{2}\right)$ [12].

\section{Some formal results}

The angular projection coefficients of Eq. (13) for, say, $g\left(r, \omega_{1}, \omega_{2}\right)$ cannot be directly compared with the standard projections typically calculated in a simulation, since these correspond to the usual spherical harmonic basis. However, it is straightforward to recover one set of coefficients from the other given the moments of the one-particle distribution function $f(x)$. For example, to determine the expansion coefficients of $u_{s s}\left(r, \omega_{1}, \omega_{2}\right)$ we need the coefficients of the rotational invariant

$$
\hat{\mathbf{s}}_{1} \cdot \hat{\mathbf{s}}_{2}=\cos \theta_{12}=\cos \theta_{1} \cos \theta_{2}+\sin \theta_{1} \sin \theta_{2} \cos \phi_{12} .
$$

From Appendix A, we have

$$
\begin{gathered}
\cos \theta=x=\langle x\rangle \mathcal{P}_{00}(x)+\sigma_{x} \mathcal{P}_{10}(x), \\
\sin \theta=\left(1-x^{2}\right)^{1 / 2}=\left[1-\left\langle x^{2}\right\rangle\right]^{1 / 2} \mathcal{P}_{11}(x),
\end{gathered}
$$

where $\sigma_{x}^{2} \equiv\left\langle x^{2}\right\rangle-\langle x\rangle^{2}$. This leads to

$$
\begin{aligned}
\hat{\mathbf{s}}_{1} \cdot \hat{\mathbf{s}}_{2}= & {\left[\langle x\rangle \mathcal{P}_{00}\left(x_{1}\right)+\sigma_{x} \mathcal{P}_{10}\left(x_{1}\right)\right] } \\
& \times\left[\langle x\rangle \mathcal{P}_{00}\left(x_{2}\right)+\sigma_{x} \mathcal{P}_{10}\left(x_{2}\right)\right] \\
& +\left[1-\left\langle x^{2}\right\rangle\right] \mathcal{P}_{11}\left(x_{1}\right) \mathcal{P}_{11}\left(x_{2}\right) \cos \phi_{12},
\end{aligned}
$$

and so [cf. Eqs. (4) and (17)]

$$
\begin{gathered}
u_{000}(r)=-\langle x\rangle^{2} J(r), \\
u_{100}(r)=u_{010}(r)=-\sigma_{x}\langle x\rangle J(r), \\
u_{110}(r)=-\sigma_{x}^{2} J(r), \\
u_{111}(r)=u_{11-1}(r)=\frac{1}{2}\left[1-\left\langle x^{2}\right\rangle\right] J(r) .
\end{gathered}
$$

Similarly, the (unnormalized) projection of $g\left(r, \omega_{1}, \omega_{2}\right)$ onto the rotational invariant $\hat{\mathbf{s}}_{1} \cdot \hat{\mathbf{s}}_{2}$ is found to be

$$
\begin{aligned}
h^{110}(r)= & 3\left\{\langle x\rangle^{2} g_{000}(r)+\sigma_{x}\langle x\rangle\left[g_{100}(r)+g_{010}(r)\right]\right. \\
& \left.+\sigma_{x}^{2} g_{110}(r)-\left[1-\left\langle x^{2}\right\rangle\right] g_{111}(r)\right\} .
\end{aligned}
$$


For an isotropic system, with $\langle x\rangle=0$ and $\left\langle x^{2}\right\rangle=1 / 3$, this reduces to the usual $h^{110}(r)=g_{110}(r)-2 g_{111}(r)$.

We can further compute the complete magnetic and thermodynamic properties of a Heisenberg spin fluid in a uniform magnetic field $\mathbf{B}_{0}$. The net magnetization in the direction $\alpha=x, y, z$ is

$$
M_{\alpha}=\mu\left\langle\sum_{j} s_{j \alpha}\right\rangle=\frac{1}{\beta} \frac{\partial \ln Z}{\partial B_{0 \alpha}}
$$

and so the magnetic susceptibility components are

$$
\begin{aligned}
\chi_{\alpha \beta} \equiv & \frac{1}{V} \frac{\partial M_{\alpha}}{\partial B_{0 \beta}}=\rho \beta \mu^{2}\left\{\left\langle s_{1 \alpha} s_{1 \beta}\right\rangle-\left\langle s_{1 \alpha}\right\rangle\left\langle s_{1 \beta}\right\rangle\right. \\
& \left.+(N-1)\left[\left\langle s_{1 \alpha} s_{2 \beta}\right\rangle-\left\langle s_{1 \alpha}\right\rangle\left\langle s_{2 \beta}\right\rangle\right]\right\} .
\end{aligned}
$$

Only the diagonal elements are nonvanishing. With $\mathbf{B}_{0}$ defining the $z$ direction, we get the longitudinal and transverse magnetic susceptibilities as

$$
\begin{aligned}
\chi_{z z} / \rho \beta \mu^{2}= & \sigma_{x}^{2}\left[1+\rho \widetilde{h}_{110}(0)\right] \\
& +\sigma_{x}\langle x\rangle\left[\rho \widetilde{h}_{100}(0)+\rho \widetilde{h}_{010}(0)\right], \\
\chi_{y y} / \rho \beta \mu^{2}= & \frac{1}{2}\left(1-\left\langle x^{2}\right\rangle\right)\left[1-\rho \widetilde{h}_{111}(0)\right] .
\end{aligned}
$$

For an isotropic system, these susceptibility expressions reduce to familiar forms.

The internal energy $U$, pressure $p$, and isothermal compressibility $K_{T}$ are calculated with the standard formulas of homogeneous fluids $[12,18]$, again thanks to the special orthogonality of the $\mathcal{Y}_{l m}(\omega)$. We have

$$
\begin{aligned}
\beta U / N= & \frac{1}{2} \frac{\rho}{(4 \pi)^{2}} \int d \mathbf{r} d \omega_{1} d \omega_{2} f\left(\omega_{1}\right) f\left(\omega_{2}\right) \\
& \times g\left(r, \omega_{1}, \omega_{2}\right) \beta u_{s s}\left(r, \omega_{1}, \omega_{2}\right) \\
= & \frac{1}{2} \rho \int d \mathbf{r} \sum_{l_{1}, l_{2}, m} g_{l_{1} l_{2} m}(r) \beta u_{l_{1} l_{2} m}(r), \\
\beta p / \rho=1 & -\frac{1}{6} \frac{\rho}{(4 \pi)^{2}} \int d \mathbf{r} d \omega_{1} d \omega_{2} f\left(\omega_{1}\right) f\left(\omega_{2}\right) \\
\times & g\left(r, \omega_{1}, \omega_{2}\right) r \frac{d \beta u\left(r, \omega_{1}, \omega_{2}\right)}{d r} \\
=1 & +\frac{2}{3} \pi \rho \sigma^{3} g_{000}(\sigma) \\
- & \frac{1}{6} \rho \int d \mathbf{r} \sum_{l_{1}, l_{2}, m} g_{l_{1} l_{2} m}(r) r \frac{d \beta u_{l_{1} l_{2} m}(r)}{d r}, \\
1 \quad \equiv & \beta\left(\frac{\partial p}{\partial \rho}\right)_{T} \\
= & 1-\frac{\rho}{(4 \pi)^{2}} \int d \mathbf{r} d \omega_{1} d \omega_{2} f\left(\omega_{1}\right) f\left(\omega_{2}\right) \\
& \times c\left(r, \omega_{1}, \omega_{2}\right)=1-\rho \tilde{c}_{000}(0) .
\end{aligned}
$$

Finally, an expression for the Helmholtz free energy $F$ is given in Appendix B.

\section{SIMULATION DETAILS}

We have used canonical ensemble (NVT) Monte Carlo simulations to calculate the thermodynamic properties in the ferromagnetic fluid and Gibbs ensemble Monte Carlo simulations $[22,23]$ to determine the gas-liquid coexistence properties.

To evaluate the free energy in the orientationally ordered phase we have chosen as a reference state the one in which the spins are perfectly aligned. Such a state is achieved by coupling the spins to an infinitely strong external field. Its free energy $F_{p}$ is easily obtained by thermodynamic integration [24] from the known free energy of the hard sphere fluid [25].

The free energy of the system in a constant field of strength $\lambda$ is then given by $[26,27]$

$$
\begin{aligned}
F_{\lambda}= & F_{p}+F_{\lambda}^{\mathrm{id}}+\int_{\lambda}^{\infty} d \lambda^{\prime} \\
& \times\left[\left\langle\sum_{i} \cos \theta_{i}\right\rangle_{\lambda^{\prime}}-\left\langle\sum_{i} \cos \theta_{i}\right\rangle_{\lambda^{\prime}}^{\mathrm{id}}\right] .
\end{aligned}
$$

Here $\langle\cdot\rangle_{\lambda}$ and $\langle\cdot\rangle_{\lambda}^{\text {id }}$ denote averaging over the system with the Hamiltonians

$$
\begin{gathered}
H_{\lambda}=H_{0}-\lambda \sum_{i} \cos \theta_{i}, \\
H_{\lambda}^{\mathrm{id}}=-\lambda \sum_{i} \cos \theta_{i},
\end{gathered}
$$

respectively, where $H_{0}$ is the Hamiltonian of the system in the absence of the field while $H_{\lambda}^{\text {id }}$ is the Hamiltonian of the ideal spin system, with $\theta_{i}$ the angle of spin $i$ with the field. The free energy $F_{\lambda}^{\text {id }}$ of the ideal spin system is given by

$$
\frac{\beta F_{\lambda}^{\mathrm{id}}}{N}=-\ln \left(4 \pi \frac{\sinh \beta \lambda}{\beta \lambda}\right) .
$$

An accurate numerical evaluation of Eq. (43) needs some care [26,27]. For the details we refer to Ref. [27], where free energy results for the liquid and solid phases of the Heisenberg fluid are reported in conjunction with a determination of the liquid-solid coexistence properties.

GEMC calculations involved a total of 512 particles and were made in cycles, each cycle comprising translationrotation trial moves of the $N$ particles, some 200 trial insertions, and 1 trial volume change. Spins were inserted preferentially parallel to the local field and the bias corrected for in the acceptance probability $[7,28]$.

\section{COMPUTED RESULTS}

We have obtained the distribution functions $f(\omega)$ and $g\left(r, \omega_{1}, \omega_{2}\right)$ for the Heisenberg spin fluid in a magnetic field $\mathbf{B}_{0}$ using the reference-hypernetted chain (RHNC) closure [29] and a reference version of the Zerah-Hansen (RZH) closure [30]; for $r>\sigma$, these are 


$$
\begin{aligned}
c^{\mathrm{RHNC}}\left(r, \omega_{1}, \omega_{2}\right)= & \exp \left[-\beta u_{s s}\left(r, \omega_{1}, \omega_{2}\right)+\gamma\left(r, \omega_{1}, \omega_{2}\right)\right. \\
& \left.+b_{\mathrm{HS}}\left(r ; \sigma_{\mathrm{ref}}\right)\right]-1-\gamma\left(r, \omega_{1}, \omega_{2}\right), \\
c^{\mathrm{RZH}}\left(r, \omega_{1}, \omega_{2}\right)= & \left\{\operatorname { e x p } \left(m ( r ) \left[-\beta u_{s s}\left(r, \omega_{1}, \omega_{2}\right)\right.\right.\right. \\
& \left.\left.\left.+\gamma\left(r, \omega_{1}, \omega_{2}\right)+b_{\mathrm{HS}}(r ; \sigma)\right]\right)-1\right\} / m(r) \\
& -\gamma\left(r, \omega_{1}, \omega_{2}\right) .
\end{aligned}
$$

The exact relation $c\left(r, \omega_{1}, \omega_{2}\right)=-1-\gamma\left(r, \omega_{1}, \omega_{2}\right)$ holds for $r<\sigma$ in both cases.

The parameter $\sigma_{\text {ref }}$ in the hard sphere bridge function $b_{\mathrm{HS}}\left(r ; \sigma_{\text {ref }}\right)$ of the RHNC equation can be treated as an adjustable hard sphere diameter [31] and chosen to minimize the free energy [32], which improves the internal consistency of this closure. In the present applications, there is little change in such optimized results compared to the simple $\sigma_{\text {ref }}=\sigma$ expedient, except for the virial pressure at the phase boundaries. However, including the optimization for the number of state points needed to determine the phase equilibrium is needlessly burdensome for our purposes at this stage and we have restricted the reported results to the nonoptimized version of the RHNC equation with $\sigma_{\text {ref }}=\sigma$. The RZH closure features a mixing function $m(r)$ $=1-\exp (-\alpha r)$ with a parameter $\alpha$ that is fixed by requiring consistency between the virial and compressibility bulk moduli [30]. (However, see below for the case $B_{0} \rightarrow 0$ ). The reference hard sphere bridge function $b_{\mathrm{HS}}(r ; \sigma)$, added by us in Eq. (48) to the usual ZH closure [30], ensures the RZH closure yields good hard sphere results in the limit of small $K$ and improves the thermodynamic results overall.

To be consistent with earlier work $[5,6]$, we have truncated the spin-spin interaction at $r=r_{c} \equiv 2.5 \sigma$; this hardly affects the thermodynamics (aside from the pressure) but does considerably raise the critical $K$ value. (Some results for the untruncated potential obtained using density functional theory and Monte Carlo simulation can be found in Refs. [7] and [8].) Because of the discontinuity of the potential at $r=r_{c}$, the pressure equation picks up an additional term; Eq. (41) now reads

$$
\begin{aligned}
\beta p / \rho= & 1+\frac{2}{3} \pi \rho \sigma^{3} g_{000}(\sigma) \\
& -\frac{2}{3} \pi \rho \int_{0}^{r_{c}} d r r^{2} g_{l_{1} l_{2} m}(r) r \frac{d \beta u_{l_{1} l_{2} m}(r)}{d r} \\
& +\frac{2}{3} \pi \rho r_{c}^{3} \sum_{l_{1}, l_{2}, m} \bar{g}_{l_{1} l_{2} m}\left(r_{c}\right) \beta u_{l_{1} l_{2} m}\left(r_{c}^{-}\right),
\end{aligned}
$$

where $\bar{g}\left(r_{c}\right) \equiv\left[g\left(r_{c}^{-}\right)+g\left(r_{c}^{+}\right)\right] / 2$.

The first set of calculations is for $\kappa=1, \rho \sigma^{3}=0.7, K$ $=1 / T^{*}=0-0.5$, and two values of the external field, $\beta \mu B_{0}=1$ and 0 , using the RZH closure. As noted earlier, we find for these cases that the one-body orientational distribution function $f(x)$ continues to be well described by the functional form of $f_{0}(x)$, but with an effective field $B$,

$$
f(x)=\frac{e^{\beta \mu B x}}{\sinh (\beta \mu B) / \beta \mu B} .
$$

In the limit $B_{0} \rightarrow 0$, the effective field $B$ is zero for $K<K_{c}$ and finite for $K>K_{c}$, where $K_{c}=1 / T_{c}^{*}$ is then the computed Curie point. This zero field limit has recently been the focus of several works [5-8], but until now no theoretical approximation has been able to completely describe the ordered phase having spontaneous magnetization.

For $K>K_{c}$, there is a singularity when $B_{0} \rightarrow 0$ that is different from the second order paramagnetic-ferromagnetic transition at $K=K_{c}$. In fact, the $B_{0}=0$ line corresponds in the $B_{0}-M$ plane (where $M$ is the magnetization per particle) to the spinodal line that indicates the equilibrium between phases with positive and negative magnetization. Consequently, the transverse susceptibility $\chi_{y y}$ (but not the longitudinal component $\chi_{z z}$ ) will diverge [33] as $B_{0} \rightarrow 0$, reflecting the negligible cost of rotating an ordered sample in the absence of an external field. It turns out that the optimization of $\alpha$ in Eq. (48) to achieve thermodynamic consistency leads to a $\chi_{y y}$ that diverges at small but nonzero fields. This deficiency is easily cured if one relaxes the thermodynamic consistency requirement and instead optimizes the $\alpha$ parameter by displacing the divergence of $\chi_{y y}$ to the $B_{0}=0$ line. We find that the thermodynamics is hardly affected by the new choice of $\alpha$.

Using Monte Carlo simulation, Nijmeijer and Weis [5] found that the paramagnetic-ferromagnetic transition for $\rho \sigma^{3}=0.7$ with the truncated potential occurs at $K_{c}=0.264$ \pm 0.001 . Our calculation in the paramagnetic phase and zero field limit yields a divergence in $\chi_{z z}$ at $K_{c}^{l}=0.2645$, in excellent agreement with the simulation value.

In Fig. 2 we show the values of the magnetization per particle $M \equiv M_{z}=\langle\cos \theta\rangle$ and the second order parameter $S$ $=\left\langle P_{2}(\cos \theta)\right\rangle$ obtained from the theory and from a standard NVT Monte Carlo simulation using 864 (for $B_{0}=1$ ), 1372 and 2048 particles (for $B_{0}=0$ ), and averages over 40000 configurations. Size effects are noticeable for $B_{0}=0$ close to and below $\left(K<K_{c}\right)$ the critical point. We include in Fig. 2 some zero-field results from density functional theory in the modified mean field approximation [8]. In the vicinity of the critical temperature, one encounters convergence difficulties in solving the integral equation as spin-spin correlations become long ranged. [Both $\chi_{z z}$ and $\chi_{y y}$ in Eqs. (38) and (39) diverge, with $\chi_{z z}$ exhibiting the characteristic $\lambda$-type divergence of second-order transitions.] In Fig. 3 we illustrate the increase in the range of the relevant angular correlation function, $h^{110}(r)$, as the Curie temperature is approached. The function $h^{110}(r)$, defined in Eq. (35), describes most of the orientational behavior of the spin fluid, since it is the projection of $g\left(r, \omega_{1}, \omega_{2}\right)$ onto the rotational invariant $\hat{\mathbf{s}}_{1} \cdot \hat{\mathbf{s}}_{2}$ $=\cos \theta_{12}$. From Eq. (35), we have $\lim _{r \rightarrow \infty} h^{110}(r)=3 M^{2}$, a limiting behavior that is exactly fulfilled in our calculations by both simulated and theoretical results. In the figure, we have subtracted the corresponding limiting values of the correlation functions so that the plots present the same asymptotic limit of zero for the two temperatures, which facilitates their comparison. The increase in the range of the correlations with the lowering of the temperature is readily appreciated. The agreement between theory and simulation is remarkable in both cases.

To get an estimate of the critical inverse temperature from the ordered-phase results, we fit the RZH magnetization in the vicinity of the critical temperature $(K<0.3)$ to a power law, 


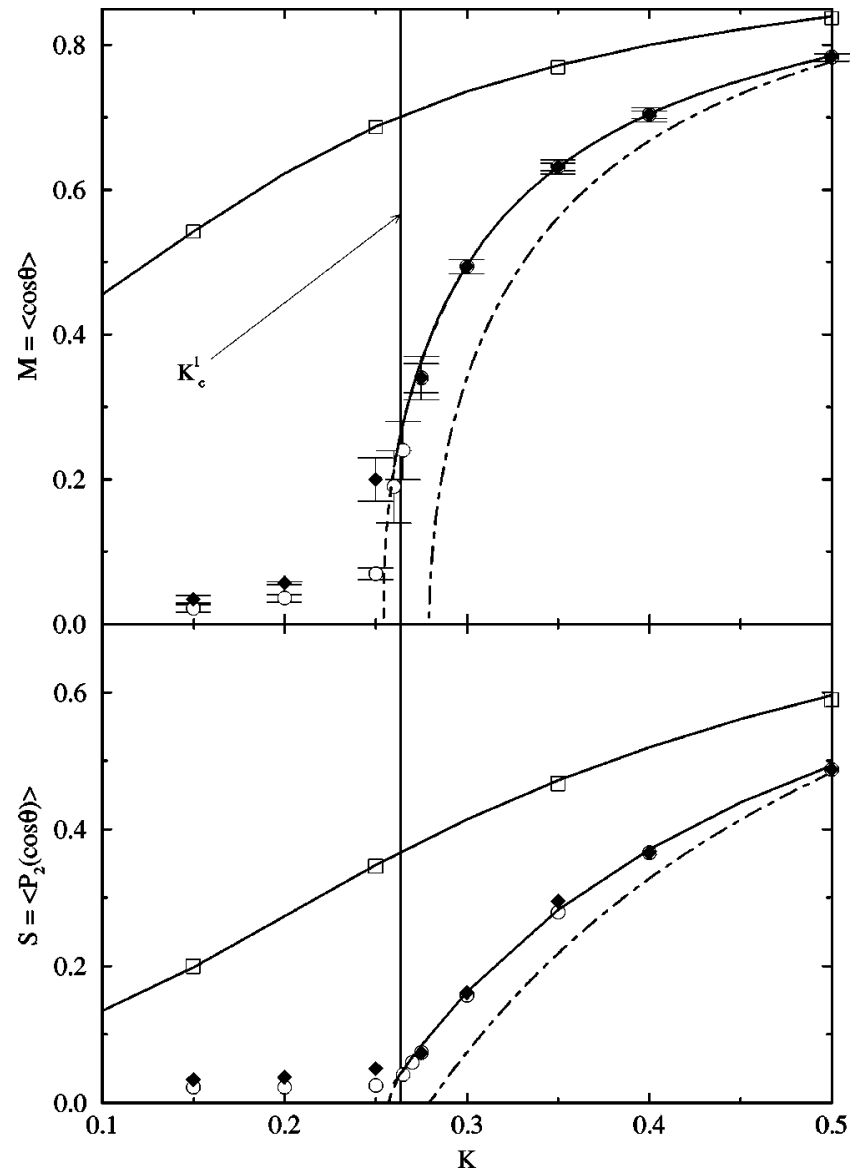

FIG. 2. Magnetization per particle $M$ and second order parameter $S$ as functions of inverse temperature $K=1 / T^{*}$ obtained from MC simulation [squares for $\beta \mu B_{0}=1$ and white circles (2048 particle sample) and black diamonds (1372 particle sample) for $\left.\beta \mu B_{0}=0\right]$. The RZH integral equation results appear as solid lines. The dashed lines correspond to a power law fit to the RZH data just above $K_{c}^{u}$. The dash-dotted curves represent density functional theory estimates [8].

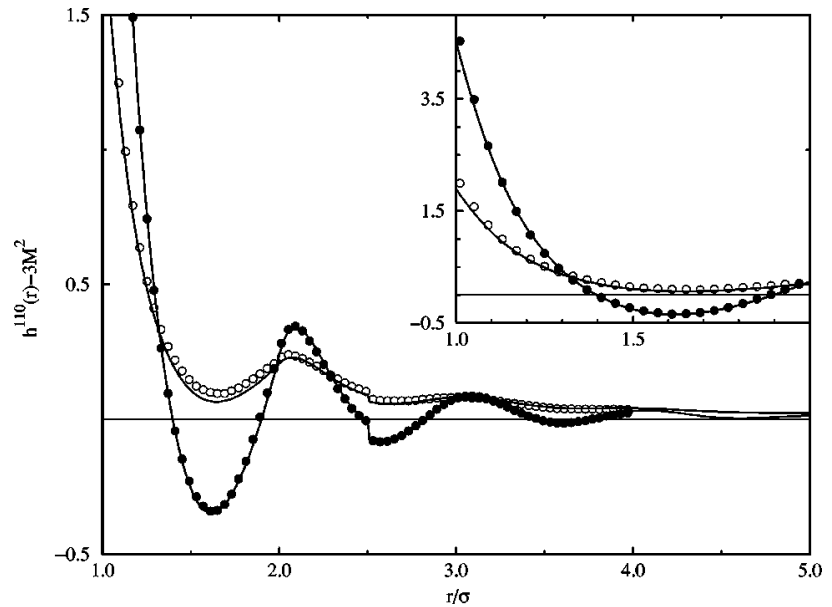

FIG. 3. Behavior of the angular correlation function $h^{110}(r)$ as the Curie temperature is approached in the ferromagnetic phase at zero field obtained from MC simulation using 1372 particles (white circles correspond to $K=0.275$ and black circles to $K=0.5$ ) for a density $\rho \sigma^{3}=0.7$. The RZH results are shown as solid lines in both cases. The long-range limiting value $3 M^{2}$ has been subtracted from both theory and simulation results to ease the comparison between the two temperatures. The discontinuity at $r=2.5 \sigma$ is due to the potential truncation.

$$
M=a\left(K-K_{c}^{u}\right)^{\beta}
$$

which leads to $a=1.694, \beta=0.397$, and $K_{c}^{u}=0.254$. The fitted value of $\beta$ is close to the value $\beta=0.387$ reported by Nijmeijer and Weis [5] and to the critical exponent of the 3D lattice Heisenberg model, $\beta=0.362 \pm 0.004$ [34] and $\beta$ $=0.3639 \pm 0.0035[35]$. However, the available data from the integral equation magnetization are too far away from $K_{c}$ to draw here any conclusion with confidence and this agreement might simply be fortuitous. Nevertheless, a similar fit carried out on the second-order parameter $S$ produces the same estimate of $K_{c}^{u}$ and so we might conclude that this is the theoretical estimate of the critical inverse temperature derived from the ordered-phase data. The agreement between

TABLE I. Magnetic and thermodynamic properties of the Heisenberg spin fluid for $\kappa=1$ and $\rho \sigma^{3}=0.7$ calculated in the RZH approximation; the values of $\chi_{y y}$ at zero field for $K>K_{c}$ correspond to the last converged solution. The MC value in parentheses is a rough estimate since, due to the vicinity of the critical point, the results at this temperature are particularly sensitive to system size. $\chi^{*} \equiv \chi / \rho \beta \mu^{2}$.

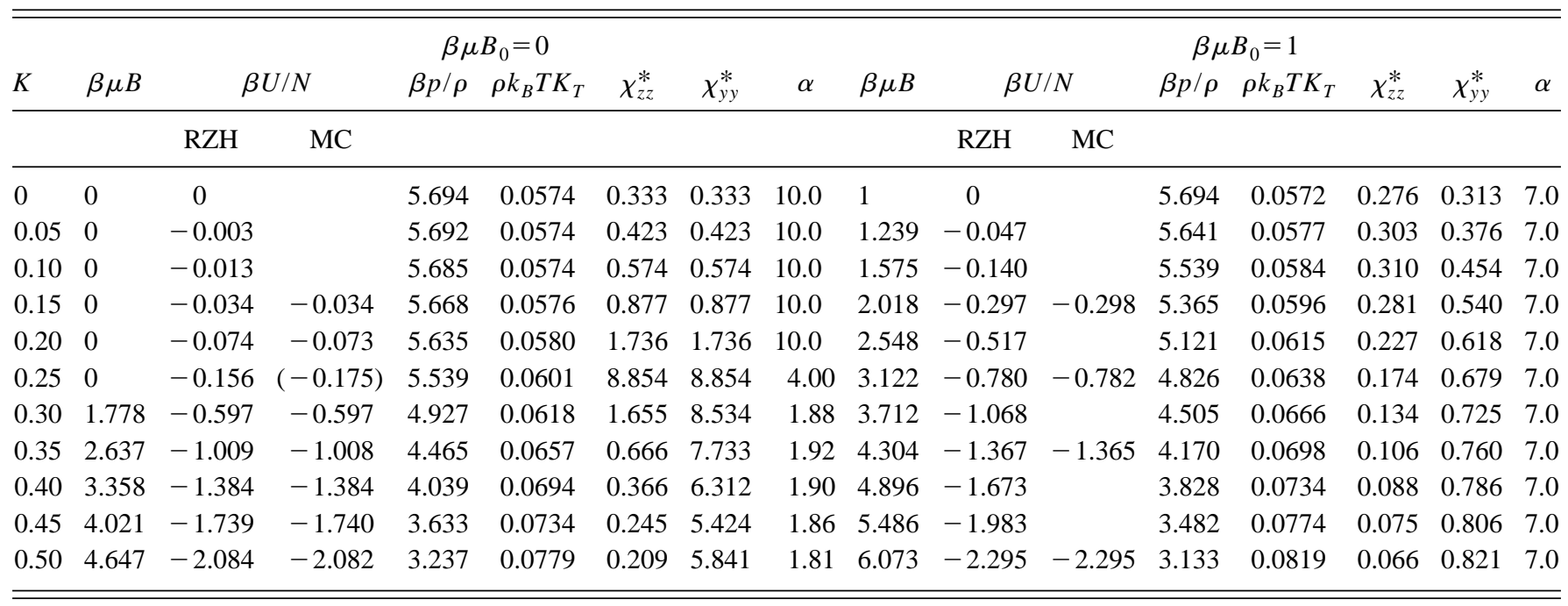




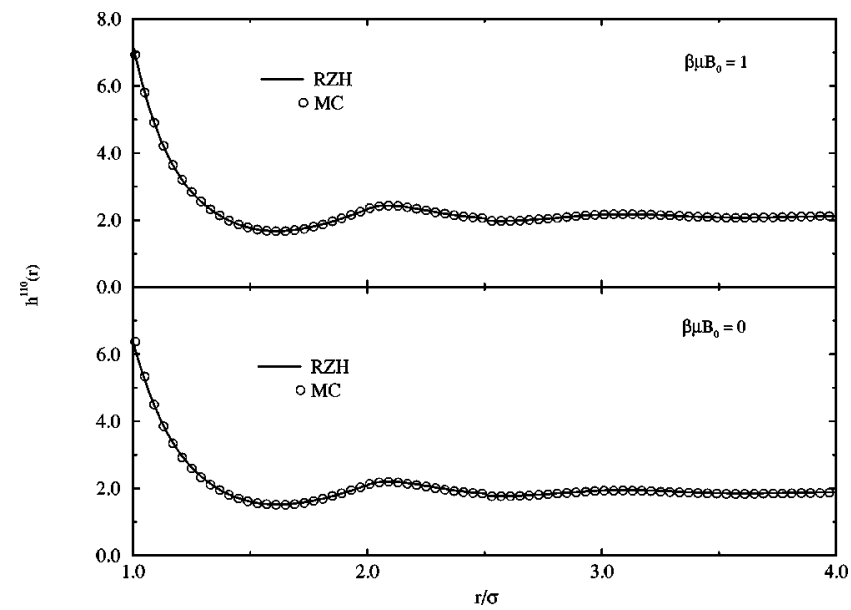

FIG. 4. The $h^{110}(r)$ angular correlation function from MC simulation (white circles) and the RZH integral equation (solid lines) for $K=0.5$ and $\rho \sigma^{3}=0.7$ with and without an external magnetic field. The discontinuity at $r=2.5 \sigma$ is due to the potential truncation.

the critical point estimates $K_{c}^{l}=0.2645$ obtained in the disordered phase and $K_{c}^{u}=0.254$ obtained in the ordered phase can be regarded as good. Clearly, there is room for improvement, perhaps by imposing further consistency conditions. In particular, one would want

$$
\chi_{z z}=\rho \mu \frac{\partial\langle x\rangle}{\partial B_{0}}
$$

to hold numerically.

The magnetic and thermodynamic properties of this first set of states, including the effective field $B$, are summarized in Table I for external fields $\beta \mu B_{0}=0$ and 1. Direct comparison with Monte Carlo data is available only for the internal energy $U$ and here we find excellent agreement with the results obtained using the RZH closure. That this excellent agreement between simulation and theory also extends to the correlation functions when the external field is turned on can be seen in Fig. 4. We note in Table I that for $\beta \mu B_{0}$ $=1$ the consistency parameter $\alpha$ in the RZH closure is insensitive to changes in magnetic interaction strength; the determining factor is the hard sphere interaction.

To get another assessment of the quality of the integral equation approximations used in this paper, we have performed a second set of calculations, with $\kappa=1$ and $\rho \sigma^{3}$ $=0.92$, for which the simulation free energies have also been calculated by means of painstaking simulation runs. For the theory, the free energy can be obtained directly from inte- grals over pair correlation functions only for the RHNC approximation (see Appendix B). In the case of the RZH approximation, we must resort to tedious thermodynamic integrations from the reference state $K=0$. Thus, our free energy estimate in this latter case is given by

$$
\frac{\beta\left(F-F_{s}^{\mathrm{id}}\right)}{N}=\frac{\beta F_{\mathrm{HS}}}{N}+\int_{0}^{K}\langle\beta U / N\rangle_{K^{\prime}} \frac{d K^{\prime}}{K^{\prime}},
$$

where $F_{\mathrm{HS}}$ is the hard sphere fluid free energy and $F_{s}^{\text {id }}$ is the noninteracting ideal spin contribution, given by

$$
\frac{\beta F_{s}^{\mathrm{id}}}{N}=-\ln \left(4 \pi \frac{\sinh \beta \mu B_{0}}{\beta \mu B_{0}}\right) \text {. }
$$

The thermodynamic integration in Eq. (53) is particularly tedious at zero field, due to the presence of the singularity at the Curie temperature and the need to optimize the $\alpha$ parameter to achieve the zero-field divergence of $\chi_{y y}$. A summary of the thermodynamic results for the new set of states is presented in Table II. Once again, theoretical results for zero field are obtained only with the RZH approximation. For finite field, the quality of the RHNC results exceeds somewhat the RZH results, in particular for the virial pressure. As to the free energy, the thermodynamic integration on the RZH internal energies yields slightly better results than the direct formula of RHNC. (If this procedure were carried out on the RHNC internal energies, the values obtained for $F$ would be practically identical, given the agreement of both approximations for the internal energy.) In any case, the RHNC direct free energies are already remarkably good. The departures observed in the RZH virial pressures at zero field stem mostly from the hard core terms in Eq. (49), since the spin-spin contributions, including the term accounting for the potential truncation, are reproduced with accuracies comparable to that of the internal energy. In order to improve these results it would be necessary to implement a thermodynamic consistency condition, which would be considerably more cumbersome at zero field, where it would be added to the enforcement of the transverse susceptibility divergence. This latter constraint is essential to reproduce the correct magnetic behavior.

Confident of the quality of our theoretical thermodynamic estimates, we are now in a position to calculate the gas-liquid phase equilibrium. Since we already have information on the zero-field phase diagram from Ref. [8], it is of primary interest to estimate the boundaries of the phase diagrams in the presence of various external magnetic fields. Thus, we also

TABLE II. Thermodynamic properties for a high density state $\left(\rho \sigma^{3}=0.92\right)$ of the hard sphere Heisenberg spin fluid. Integral equation vs MC (NVT) simulation results using 512 spins.

\begin{tabular}{cccccccccccccc}
\hline \hline & & \multicolumn{3}{c}{$\beta\left(F-F_{s}^{\text {id }}\right) / N$} & \multicolumn{3}{c}{$\beta P / \rho$} & \multicolumn{3}{c}{$\beta U / N$} & & \multicolumn{3}{c}{$M$} \\
\cline { 3 - 12 }$\beta \mu B_{0}$ & $T^{*}$ & MC & RHNC & RZH & MC & RHNC & RZH & MC & RHNC & RZH & MC & RHNC & RZH \\
\hline \multirow{2}{*}{0} & 1.0 & -1.675 & & -1.672 & 3.20 & & 2.76 & -7.642 & & -7.631 & 0.935 & & 0.933 \\
& 3.0 & 2.978 & & 2.96 & 9.65 & & 9.16 & -1.744 & & 1.742 & 0.764 & & 0.759 \\
3.38 & 1.0 & -3.380 & -3.48 & -3.38 & 2.78 & 2.91 & 2.55 & -7.824 & -7.822 & -7.819 & 0.948 & 0.948 & 0.948 \\
& 3.0 & 1.62 & 1.49 & 1.57 & 9.03 & 8.95 & 8.6 & -2.253 & -2.250 & -2.249 & 0.881 & 0.880 & 0.880 \\
\hline \hline
\end{tabular}

${ }^{a}$ Value obtained from thermodynamic integration of the excess internal energy via Eq. (53). 


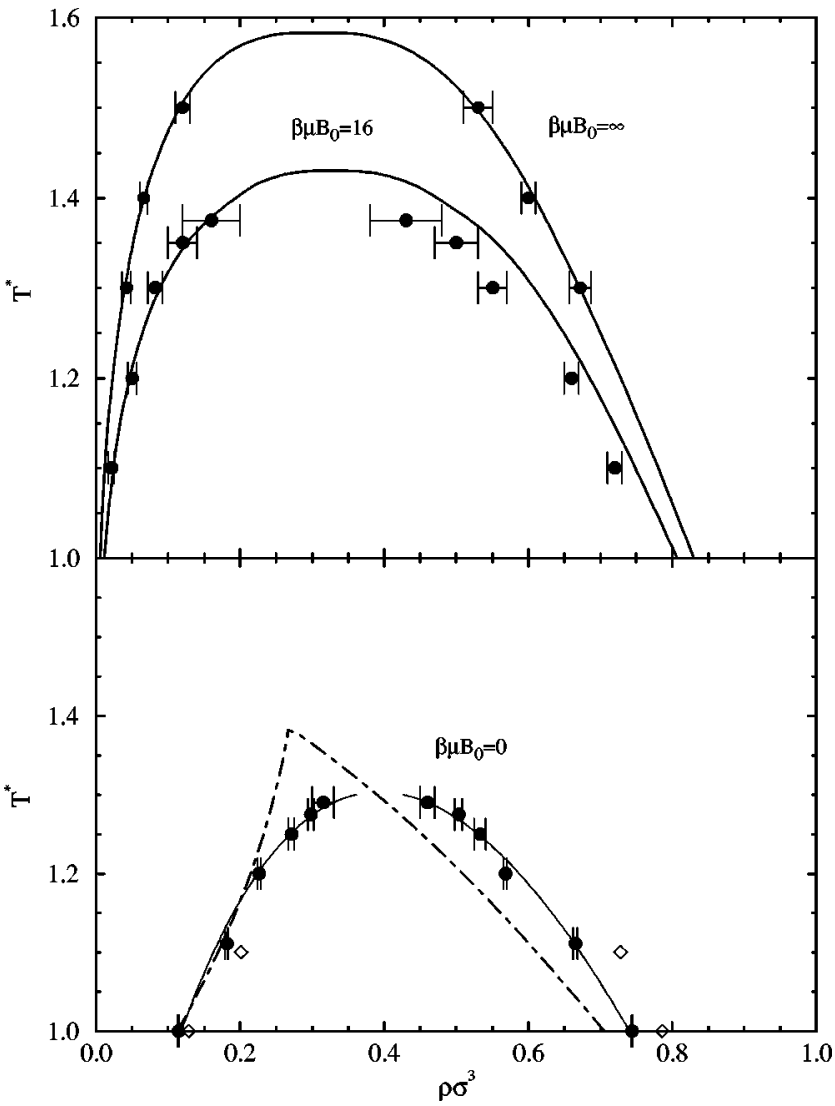

FIG. 5. Phase diagram of the Heisenberg spin fluid in the presence of an external field (upper figure) and at zero field (lower figure). Simulation data are shown as black circles and RHNC results as solid lines. In the lower figure, the simulation data are taken from Ref. [6] while the dash-dotted line indicates density functional theory results taken from Ref. [8]; white diamonds represent two equilibrium points obtained from thermodynamic integration of the RZH results.

consider a fully aligned spin system, i.e., a spin fluid under the action of an infinitely strong external field. This turns out to be nothing but a fluid of hard spheres plus attractive Yukawa potential, for which the RHNC approximation has already been shown to yield excellent results [36]. The simplicity of this extreme case makes feasible the optimization of the reference system hard sphere diameter $\sigma_{\mathrm{HS}}$ in the
RHNC calculations, whereby thermodynamic properties (and in particular the pressure and free energy) are substantially improved. The phase equilibrium is calculated by means of a double tangent construction on the free energy data furnished by the RHNC. The results of this calculation are presented in Fig. 5, along with GEMC data, which are also summarized in Table III. Comparing the pure Yukawa and the zero field results in this figure, one notes that the equilibrium densities are only slightly affected by the external field. We have therefore chosen a relatively large field, $\beta \mu B_{0}=16$, to perform an additional set of calculations at nonzero but finite field. The results obtained from the RHNC approximation, seen in Fig. 5, although relatively good, are somewhat worse than those obtained for the pure Yukawa; this is a direct consequence of the neglect of the optimization condition for finite field. The situation is slightly worse using the RZH equation in the zero field case. Here the use of thermodynamic integration based on the energy route, which yields extremely good thermodynamics, is too time consuming, since it has to be performed for every density needed to map the isotherms required for the double tangent construction. Consequently, we have used the virial route starting from low density results, which is somewhat poorer, since RZH virial pressures are not as accurate as the corresponding internal energies (see Table II). Therefore, we have only calculated two equilibrium points (each implies 100 integral equation solutions), which are shown in the lower part of Fig. 5. In this case, the data show deviations within $8-10 \%$, which compares poorly with the $1-5 \%$ deviations in the pure RHNC or $0.5 \%$ in the optimized RHNC.

Finally, both theory and simulation show that the effect of an external field on the spin system (and presumably also on dipolar fluids [37]) is a considerable increase in the critical temperature, while equilibrium densities are not significantly affected; i.e., external fields tend to stabilize the liquid phase.

In summary, we have presented an application of a novel technique to study orientationally ordered fluid phases, based on a combination of traditional integral equation methods with an appropriate choice of orthogonal polynomials to expand the correlation functions. Detailed comparisons with standard NVT Monte Carlo, finite size scaling, and GEMC show that the proposed approach is powerful enough to give a correct account of the magnetic transition and a more than

TABLE III. Gas-liquid coexistence properties for the Heisenberg spin fluid in the presence of external fields as obtained from GEMC calculations. The subscripts $g$ and $l$ denote the gas and liquid phases, respectively; $\mu$ is the chemical potential.

\begin{tabular}{|c|c|c|c|c|c|c|c|c|c|c|c|c|c|}
\hline$\beta \mu B_{0}$ & $T^{*}$ & $\begin{array}{l}\text { No. of } \\
\text { cycles }\end{array}$ & $\rho_{g}$ & & $\rho_{l}$ & $\left(\beta P \sigma^{3}\right)_{g}$ & $\left(\beta P \sigma^{3}\right)_{l}$ & $(\beta \mu)_{g}$ & $(\beta \mu)_{l}$ & $(\beta U / N)_{g}$ & $(\beta U / N)_{l}$ & $M_{g}$ & $M_{l}$ \\
\hline \multirow[t]{5}{*}{16} & 1.1 & 60000 & $0.021 \pm 0.004$ & 0.72 & \pm 0.01 & 0.019 & -0.13 & -15.48 & -15.46 & -0.181 & -5.57 & 0.935 & 0.961 \\
\hline & 1.2 & 30000 & $0.051 \pm 0.006$ & 0.66 & \pm 0.01 & 0.040 & 0.005 & -13.62 & -13.61 & -0.384 & -4.59 & 0.930 & 0.956 \\
\hline & 1.3 & 40000 & $0.082 \pm 0.01$ & 0.55 & \pm 0.02 & 0.058 & 0.022 & -12.58 & -12.58 & -0.546 & -3.43 & 0.926 & 0.948 \\
\hline & 1.35 & 40000 & $0.12 \pm 0.02$ & 0.50 & \pm 0.03 & 0.078 & 0.073 & -11.72 & -11.71 & -0.772 & -2.98 & 0.926 & 0.945 \\
\hline & 1.375 & 30000 & $0.16 \pm 0.04$ & 0.43 & \pm 0.05 & 0.094 & 0.09 & -11.90 & -11.89 & -1.10 & -2.56 & 0.928 & 0.940 \\
\hline \multirow[t]{3}{*}{$\infty$} & 1.3 & 20000 & $0.042 \pm 0.006$ & 0.672 & $2 \pm 0.015$ & 0.033 & 0.050 & -3.61 & -3.62 & -0.338 & -4.74 & & \\
\hline & 1.4 & 40000 & $0.066 \pm 0.005$ & 0.60 & \pm 0.01 & 0.049 & 0.030 & -3.49 & -3.49 & -0.491 & -3.87 & & \\
\hline & 1.5 & 20000 & $0.12 \pm 0.01$ & 0.53 & \pm 0.02 & 0.073 & 0.076 & -3.64 & -3.64 & -0.803 & -3.21 & & \\
\hline
\end{tabular}


reasonable description of the gas-liquid phase behavior. An extension of this work to dipolar systems is currently under way.

\section{ACKNOWLEDGMENTS}

F.L. thanks the Dirección General de Investigación y Desarrollo (Spain) for sabbatical support under Grant No. SAB95-0373. E.L. would like to acknowledge the institutional agreement CSIC/CNRS, which has provided funds for several visits to Orsay, where part of this work was conceived. Computing time on a CRAY C-98 was granted by the Institut de Développement et de Ressources en Informatique (IDRIS). This work was supported in part by the Dirección General de Investigación Científica y Técnica (Spain) under Grant Nos. PB94-0112 and PB94-0265. The Laboratoire de Physique Théorique et Hautes Energies is a Laboratoire associé au Center National de la Recherche Scientifique (URA 063).

\section{APPENDIX A: MODIFIED LEGENDRE FUNCTIONS}

The prerequisites [38] for construction of the modified Legendre functions are the moments

$$
\left\langle x^{k}\right\rangle \equiv \frac{1}{2} \int_{-1}^{1} d x f(x) x^{k} .
$$

For an exponential distribution

$$
f(x)=\left(\frac{\sinh \lambda}{\lambda}\right)^{-1} e^{\lambda x},
$$

these are obtained as

$$
\left\langle x^{k}\right\rangle=\left(\frac{\sinh \lambda}{\lambda}\right)^{-1} \frac{d^{k}}{d \lambda^{k}}\left(\frac{\sinh \lambda}{\lambda}\right) .
$$

Then, in a straightforward application of the Gram-Schmidt method [19], we begin with $\mathcal{P}_{00}(x)=1$ and construct the next polynomial with $m=0$ as $\mathcal{P}_{10}(x)=a(x-b)$. Orthogonality then requires

$$
\left\langle\mathcal{P}_{00}(x) \mathcal{P}_{10}(x)\right\rangle=a(\langle x\rangle-b)=0
$$

or $b=\langle x\rangle$, while normalization leads to

$$
\left\langle\mathcal{P}_{10}^{2}(x)\right\rangle=a^{2}\left[\left\langle x^{2}\right\rangle-2\langle x\rangle^{2}+\langle x\rangle^{2}\right]=1
$$

or $a=1 /\left[\left\langle x^{2}\right\rangle-\langle x\rangle^{2}\right]^{1 / 2}$. Thus we find

$$
\mathcal{P}_{10}(x)=\frac{x-\langle x\rangle}{\left[\left\langle x^{2}\right\rangle-\langle x\rangle^{2}\right]^{1 / 2}}=\frac{1}{\left(D_{00} D_{10}\right)^{1 / 2}}\left|\begin{array}{cc}
1 & \langle x\rangle \\
1 & x
\end{array}\right| .
$$

In the second equality of Eq. (A6), we have used two determinants $D_{l m}$, defined in general for $m=0,1,2, \ldots, l$, by

$$
D_{l m}=\left|\begin{array}{cccc}
\left\langle\left(1-x^{2}\right)^{m}\right\rangle & \left\langle x\left(1-x^{2}\right)^{m}\right\rangle & \cdots & \left\langle x^{l-m}\left(1-x^{2}\right)^{m}\right\rangle \\
\left\langle x\left(1-x^{2}\right)^{m}\right\rangle & \left\langle x^{2}\left(1-x^{2}\right)^{m}\right\rangle & \cdots & \left\langle x^{l-m+1}\left(1-x^{2}\right)^{m}\right\rangle \\
\cdot & \cdot & \cdots & . \\
\cdot & \cdot & \cdots & . \\
\cdot & \cdot & \cdots & . \\
\left\langle x^{l-m}\left(1-x^{2}\right)^{m}\right\rangle & \left\langle x^{l-m+1}\left(1-x^{2}\right)^{m}\right\rangle & \cdots & \left\langle x^{2(l-m)}\left(1-x^{2}\right)^{m}\right\rangle
\end{array}\right|,
$$

which we supplement with $D_{m-1, m} \equiv 1$. The general expression for $\mathcal{P}_{l 0}(x)$ is given by Akhiezer [38] as

$$
\mathcal{P}_{l 0}(x)=\frac{1}{\left(D_{l-1,0} D_{l 0}\right)^{1 / 2}}\left|\begin{array}{cccc}
1 & \langle x\rangle & \cdots & \left\langle x^{l}\right\rangle \\
\langle x\rangle & \left\langle x^{2}\right\rangle & \cdots & \left\langle x^{l+1}\right\rangle \\
\cdot & \cdot & \cdots & . \\
\cdot & . & \cdots & . \\
\cdot & . & \cdots & . \\
\left\langle x^{l-1}\right\rangle & \left\langle x^{l}\right\rangle & \cdots & \left\langle x^{2 l-1}\right\rangle \\
1 & x & \cdots & x^{l}
\end{array}\right| .
$$

Retaining the general structure of the standard $P_{l m}(x)$, a similar procedure is followed for higher $m$ values. Thus, the sequence for $m=1$ begins with $\mathcal{P}_{11}(x)=\left(1-x^{2}\right)^{1 / 2} /\left(1-\left\langle x^{2}\right\rangle\right)^{1 / 2} ; \mathcal{P}_{21}(x)=\left(1-x^{2}\right)^{1 / 2} a(x-b)$ is then constructed to be orthogonal to $\mathcal{P}_{11}(x)$ and normalized to unity. More briefly, we can generalize the expression given by Akhiezer for $\mathcal{P}_{l 0}(x)$ and define finally 


$$
\mathcal{P}_{l m}(x)=\frac{\left(1-x^{2}\right)^{m / 2}}{\left(D_{l-1, m} D_{l m}\right)^{1 / 2}}\left|\begin{array}{cccc}
\left\langle\left(1-x^{2}\right)^{m}\right\rangle & \left\langle x\left(1-x^{2}\right)^{m}\right\rangle & \cdots & \left\langle x^{l-m}\left(1-x^{2}\right)^{m}\right\rangle \\
\left\langle x\left(1-x^{2}\right)^{m}\right\rangle & \left\langle x^{2}\left(1-x^{2}\right)^{m}\right\rangle & \cdots & \left\langle x^{l-m+1}\left(1-x^{2}\right)^{m}\right\rangle \\
\cdot & \cdot & \cdots & . \\
\cdot & \cdot & \cdots & . \\
\cdot & \cdot & \cdots & . \\
\left\langle x^{l-m-1}\left(1-x^{2}\right)^{m}\right\rangle & \left\langle x^{l-m}\left(1-x^{2}\right)^{m}\right\rangle & \cdots & \left\langle x^{2(l-m)-1}\left(1-x^{2}\right)^{m}\right\rangle \\
1 & x & \cdots & x^{l-m}
\end{array}\right| .
$$

Integrals over $x=\cos \theta$ are evaluated using Gaussian quadrature [38] based on the $n$ zeroes $x_{k}$ of $\mathcal{P}_{n 0}(x)$,

$$
\frac{1}{2} \int_{-1}^{1} d x f(x) h(x) \approx \sum_{k=1}^{n} w_{k} h\left(x_{k}\right),
$$

with the weights

$$
w_{k}=\left[\sum_{l=0}^{n-1} \mathcal{P}_{l 0}^{2}\left(x_{k}\right)\right]^{-1} .
$$

Equation (A10) is exact if $h(x)$ is a polynomial of $x$ of degree $2 n-1$ or less. This ensures the exact numerical orthonormality of the polynomials used in the calculation,

$$
\sum_{k=1}^{n} w_{k} \mathcal{P}_{l m}\left(x_{k}\right) \mathcal{P}_{l^{\prime} m}\left(x_{k}\right)=\delta_{l l^{\prime}}
$$

for $l, l^{\prime}<n$ [cf. Eq. (14)].

\section{APPENDIX B: HELMHOLTZ FREE ENERGY}

The Helmholtz free energy $F^{\text {id }}=-k_{B} T \ln Z^{\text {id }}$ of the ideal spin gas is, from Eq. (3),

$$
\frac{\beta F^{\mathrm{id}}}{N}=\ln \rho \Lambda^{3}-1-\ln \left(4 \pi \frac{\sinh \beta \mu B_{0}}{\beta \mu B_{0}}\right) .
$$

To calculate the total free energy we use the familiar " charging' process, turning on the spin-spin interactions with a parameter $\xi, 0 \leqslant \xi \leqslant 1$. However, as emphasized by Sullivan [39], the one-body distribution $f(\omega)$ should remain unchanged as the interaction is turned on. Thus we will also adopt an effective external field $\mathcal{B}_{0}(\xi)$ designed to maintain fixed the $f(\omega)$ found by calculation, Eq. (50). Define then the partition function

$$
\begin{aligned}
Z(\xi)= & \frac{1}{N ! \Lambda^{3 N}} \int \prod_{j=1}^{N}\left[d \mathbf{r}_{j} d \omega_{j}\right] \exp \left(\beta \mu \mathcal{B}_{0}(\xi) \sum_{j} \cos \theta_{j}\right. \\
& \left.-\beta \sum_{i<j} u_{0}\left(r_{i j}\right)-\xi \beta \sum_{i<j} u_{s s}\left(r_{i j}, \omega_{i}, \omega_{j}\right)\right)
\end{aligned}
$$

where the external field is such that

$$
\begin{gathered}
\mathcal{B}_{0}(\xi=0)=B, \\
\mathcal{B}_{0}(\xi=1)=B_{0} .
\end{gathered}
$$

Then from $\beta F(\xi)=-\ln Z(\xi)$ we get

$$
\begin{aligned}
\frac{d \beta F(\xi)}{d \xi}= & -N \beta \mu \frac{d \mathcal{B}_{0}(\xi)}{d \xi}\langle x\rangle+\frac{1}{2} \frac{N \rho}{(4 \pi)^{2}} \int d \mathbf{r} d \omega_{1} d \omega_{2} \\
& \times f\left(\omega_{1}\right) f\left(\omega_{2}\right) g\left(r, \omega_{1}, \omega_{2} \mid \xi\right) \beta u_{s s}\left(r, \omega_{1}, \omega_{2}\right),
\end{aligned}
$$

where $g\left(r, \omega_{1}, \omega_{2} \mid \xi\right)$ is the pair distribution function for the partially charged system. We note that $F(0)$ is the free energy of hard spheres with noninteracting spins in an external field $\mathcal{B}_{0}(0)$; that is,

$$
\frac{\beta F(0)}{N}=\frac{\beta F_{\mathrm{HS}}}{N}-\ln \left(4 \pi \frac{\sinh \beta \mu B}{\beta \mu B}\right),
$$

where $F_{\mathrm{HS}}$ is the total free energy of a hard sphere system. Integration of Eq. (B3) then gives

$$
\begin{aligned}
\frac{\beta F}{N}= & \frac{\beta F(0)}{N}-\beta \mu\left[\mathcal{B}_{0}(1)-\mathcal{B}_{0}(0)\right]\langle x\rangle+\frac{\beta \Delta F}{N} \\
= & \frac{\beta F_{\mathrm{HS}}}{N}-\ln \left(4 \pi \frac{\sinh \beta \mu B}{\beta \mu B}\right) \\
& +\beta \mu\left(B-B_{0}\right)\langle x\rangle+\frac{\beta \Delta F}{N},
\end{aligned}
$$

where the spin-spin contribution is

$$
\begin{aligned}
\frac{\beta \Delta F}{N} \equiv & \frac{1}{2} \frac{\rho}{(4 \pi)^{2}} \int d \mathbf{r} d \omega_{1} d \omega_{2} f\left(\omega_{1}\right) f\left(\omega_{2}\right) \\
& \times \int_{0}^{1} d \xi g\left(r, \omega_{1}, \omega_{2} \mid \xi\right) \beta u_{s s}\left(r, \omega_{1}, \omega_{2}\right) .
\end{aligned}
$$

Finally, the excess free energy $F^{\mathrm{ex}}=F-F^{\mathrm{id}}$ is

$$
\begin{aligned}
\frac{\beta F^{\mathrm{ex}}}{N}= & {\left[\beta \mu B\langle x\rangle-\ln \left(\frac{\sinh \beta \mu B}{\beta \mu B}\right)\right] } \\
& -\left[\beta \mu B_{0}\langle x\rangle-\ln \left(\frac{\sinh \beta \mu B_{0}}{\beta \mu B_{0}}\right)\right] \\
& +\frac{\beta F_{\mathrm{HS}}^{e x}}{N}+\frac{\beta \Delta F}{N},
\end{aligned}
$$

with $\beta F_{\mathrm{HS}}^{\mathrm{ex}} / N=\beta F_{\mathrm{HS}} / N-\ln \rho \Lambda^{3}+1$ the excess free energy of the hard sphere system. 
The final step is evaluation of $\beta \Delta F / N$. We follow the derivation of Morita and Hiroike [40]; appropriate generalizations for molecular systems [20] and special polynomials [41] have already been described. The result is

$$
\begin{aligned}
\frac{\beta \Delta F}{N}= & -\frac{1}{2} \rho \tilde{c}_{000}(0)-\frac{1}{2 \rho} \int \frac{d \mathbf{k}}{(2 \pi)^{3}} \sum_{m}\left\{\ln \operatorname{det}\left[I+(-1)^{m} \rho \widetilde{H}_{m}(k)\right]-(-1)^{m} \rho \operatorname{tr}\left[\widetilde{H}_{m}(k)\right]+\frac{1}{2} \rho^{2} \operatorname{tr}\left[\widetilde{C}_{m}^{2}(k)-\widetilde{\Gamma}_{m}^{2}(k)\right]\right\} \\
& +\frac{1}{2} \rho \tilde{c}_{\mathrm{HS}}(0)+\frac{1}{2 \rho} \int \frac{d \mathbf{k}}{(2 \pi)^{3}}\left\{\ln \left[1+\rho \tilde{h}_{\mathrm{HS}}(k)\right]-\rho \widetilde{h}_{\mathrm{HS}}(k)+\frac{1}{2} \rho^{2}\left[\widetilde{c}_{\mathrm{HS}}^{2}(k)-\widetilde{\gamma}_{\mathrm{HS}}^{2}(k)\right]\right\} \\
& +\frac{1}{2} \rho \int d \mathbf{r} \int_{0}^{1} d \xi \sum_{l_{1}, l_{2}, m} g_{l_{1} l_{2} m}(r \mid \xi) \frac{\partial b_{l_{1} l_{2} m}(r \mid \xi)}{\partial \xi}
\end{aligned}
$$

where $\widetilde{\Gamma}_{m}(k), \widetilde{C}_{m}(k)$, and $\widetilde{H}_{m}(k)$ are symmetric matrices with elements $\tilde{\gamma}_{l_{1} l_{2} m}(k), \widetilde{c}_{l_{1} l_{2} m}(k)$, and $\widetilde{h}_{l_{1} l_{2} m}(k)$, respectively, for $l_{1}, l_{2} \geqslant m$, with $h=g-1$; det and tr are the determinant and trace operations. The last term of Eq. (B8) must be approximated. For the RHNC closure, one assumes the bridge function does not change appreciably as the interaction goes from the hard sphere potential to the full potential and the last term is neglected.

[1] N. D. Mermin, Phys. Rev. 137, A1441 (1965).

[2] J. R. Henderson, in Fundamentals of Inhomogeneous Fluids, edited by D. Henderson (Dekker, New York, 1992), Chap. 2.

[3] A preliminary account of the procedure has been given earlier. See F. Lado and E. Lomba, Phys. Rev. Lett. 80, 3535 (1998).

[4] J. S. Høye and G. Stell, Phys. Rev. Lett. 36, 1569 (1976).

[5] M. J. P. Nijmeijer and J. J. Weis, Phys. Rev. Lett. 75, 2887 (1995); Phys. Rev. E 53, 591 (1996).

[6] J. J. Weis, M. J. P. Nijmeijer, J. M. Tavares, and M. M. Telo da Gama, Phys. Rev. E 55, 436 (1997).

[7] E. Lomba, J. J. Weis, N. G. Almarza, F. Bresme, and G. Stell, Phys. Rev. E 49, 5169 (1994).

[8] J. M. Tavares, M. M. Telo da Gama, P. I. C. Teixeira, J. J. Weis, and M. J. P. Nijmeijer, Phys. Rev. E 52, 1915 (1995).

[9] See G. S. Cargill II and R. W. Cochrane, in Amorphous Magnetism, edited by H. O. Hooper and A. M. de Graaf (Plenum, New York, 1973), p. 313.

[10] G. Bush and H. J. Guentherodt, Phys. Lett. 27A, 110 (1968); B. Kraeft and H. Alexander, Phys. Kondens. Mater. 16, 281 (1973).

[11] T. Albrecht, C. Bührer, M. Fähnle, K. Maier, D. Platzek, and J. Reske, Appl. Phys. A: Mater. Sci. Process. 65, 215 (1997).

[12] J. P. Hansen and I. R. McDonald, Theory of Simple Liquids (Academic, London, 1986).

[13] D. Henderson, in Fundamentals of Inhomogeneous Fluids, edited by D. Henderson (Dekker, New York, 1992), Chap. 4.

[14] See, for example, T. L. Hill, Statistical Mechanics (McGrawHill, New York, 1956), Chap. 6.

[15] R. Lovett, C. Y. Mou, and F. P. Buff, J. Chem. Phys. 65, 570 (1976)

[16] M. Wertheim, J. Chem. Phys. 65, 2377 (1976).

[17] F. Lado, Phys. Rev. E 55, 426 (1997).

[18] C. G. Gray and K. E. Gubbins, Theory of Molecular Fluids (Clarendon, Oxford, 1984), Vol. 1.

[19] See, for example, G. Arfken, Mathematical Methods for Physicists (Academic, Orlando, 1985), Chap. 9.
[20] F. Lado, Mol. Phys. 47, 283 (1982).

[21] Since the interatomic vector $\mathbf{r}_{12}$ is completely decoupled from the spin orientations $\hat{\mathbf{s}}_{1}, \hat{\mathbf{s}}_{2}$ in the Heisenberg fluid, the solution of the $\mathrm{OZ}$ plus closure equations here is actually simpler than that described in Ref. [20].

[22] A. Panagiotopoulos, Mol. Simul. 9, 1 (1992).

[23] B. Smit, in Computer Simulation in Chemical Physics (Kluwer, Dordrecht, 1993), edited by M. P. Allen and D. J. Tildesley.

[24] See, for example, M. P. Allen and D. J. Tildesley, Computer Simulation of Liquids (Clarendon, Oxford, 1987).

[25] N. F. Carnahan and K. E. Starling, J. Chem. Phys. 51, 635 (1969).

[26] J. A. C. Veerman and D. Frenkel, Phys. Rev. A 41, 3237 (1990).

[27] E. Lomba, J. J. Weis, and C. F. Tejero, Phys. Rev. E 58, 3426 (1998).

[28] J. M. Caillol, J. Chem. Phys. 98, 9835 (1993).

[29] F. Lado, Phys. Rev. A 8, 2548 (1973).

[30] G. Zerah and J. P. Hansen, J. Chem. Phys. 84, 2336 (1986).

[31] Y. Rosenfeld and N. W. Ashcroft, Phys. Rev. A 20, 1208 (1979).

[32] F. Lado, Phys. Lett. 89A, 196 (1982).

[33] L. D. Landau, E. M. Lifshitz, and L. P. Pitaevskii, Electrodynamics of Continuous Media (Pergamon, Oxford, 1984), 2nd Ed. Sec. 46.

[34] C. Holm and W. Janke, Phys. Rev. B 48, 936 (1993).

[35] K. Chen, A. M. Ferrenberg, and D. P. Landau, Phys. Rev. B 48, 3249 (1993).

[36] E. Lomba and N. G. Almarza, J. Chem. Phys. 100, 8367 (1994).

[37] M. J. Stevens and G. S. Grest, Phys. Rev. E 51, 5962 (1995).

[38] N. I. Akhiezer, The Classical Moment Problem (Hafner, New York, 1965), Chap. 1.

[39] D. E. Sullivan, Phys. Rev. A 25, 1669 (1982).

[40] T. Morita and K. Hiroike, Prog. Theor. Phys. 23, 1003 (1960).

[41] F. Lado, Phys. Rev. E 54, 4411 (1996). 